http://jmscr.igmpublication.org/home/ ISSN (e)-2347-176x ISSN (p) 2455-0450 crossref DOI: https://dx.doi.org/10.18535/jmscr/v8i9.45

\title{
Somapacitan-Beco: For Adult Human Growth Hormone Deficiency
}

\author{
Author \\ Harichandana Punukula ${ }^{1}$
}

Pharm D, Bharat Institute of Technology, Hyderabad, Telangana, India

\begin{abstract}
Somapacitan (Sogroya) is the first human hormone of growth (hGH) therapy that is only administered once a week by injection under the skin; other FDA-approved hGH formulations must be given daily to adults with growth hormone deficiency. Somapacitan in adults with a growth hormone deficit (GHD) is suggested for the replacement of endogenous growth hormone.

GHD is a disorder in which the body contains not enough growth hormone alone. Growth hormone controls many functions in the body, including fat storage in the trunk or central region of the body, and is related to severe medical conditions. Here we are presenting side effects, limitations and the therapeutic efficacy of somapacitan in accomplishing the human growth harmone deficiency.

Keywords: Somapacitan, human growth factor, Sogroya.
\end{abstract}

\section{Introduction:}

Somapacitan is a growth hormone drug, marketed under the Sogroya brand name. Somapacitan is similar to a human growth hormone. Somapacitan-beco is created by recombinant DNA technology in Escherichia coli.

Back pain, joint paint, indigestion, sleep issues, swelling, tonsillities, arms or lower legs swelling, vomiting, insufficient adrenaline, high blood pressure, rise in blood creatine phosphokinase (a form in enzyme) and anaemia are the most common side-effects ${ }^{1}$.

\section{Mechanism of Action}

Somapacitan is a long term derivative of a human growth hormone developed to bind to albumin. It is based on protéin technology used for formulating long-acting insulins and glucagon-like peptide- $1^{2}$.

\section{Adult Growth Hormone Deficiency}

The pituitary gland produces growth hormone. GHD can affect the body of adults in many respects including changes in the composition of the body, lipids, metabolism of carbohydrates, bones, and muscles. In the United States, more than 50,000 people are GHD diagnosed each year with nearly 6,000 new patients ${ }^{2}$.

\section{Sogroya}

The $10 \mathrm{mg} / 1.5 \mathrm{~mL}(6.7 \mathrm{mg} / \mathrm{mL})$ injection of Sogroya (somapacitan-beco) has been prescription medication which contains the body's human growth hormone and which is used to treat adults who do not produce sufficient growth hormones. It is uncertain if Sogroya in children is safe and reliable ${ }^{3}$. 


\section{Safety Information:}

Sogroya is not used when:

- You are reported for certain kinds of cardiac or stomach operation, trauma or difficulties of breathing

- Cancer or other tumours are present.

- Somapacitan-beco or any of Sogroya's ingredients are allergic

- The health care professional tells you that you have certain forms of diabetes-induced eye issues.

Prior to Sogroya administration Health care provider should be informed if you:

- have had heart or stomach surgery, trauma or serious breathing problems, cancer or any tumor, diabetes, adrenal gland problems, taking replacement therapy with glucocorticoids, thyroid gland problems, liver problems and are pregnant or planing to become pregnant. It is unknown if Sogroya will harm your unborn baby. Breastfeeding or planing to breastfeed should also be informed to your health care provider. It is unknown if Sogroya passes into your breast milk.

Healthcare provider should be informed about all the medicines you take, including prescription and over-the-counter medicines, vitamins, and herbal supplements. Sogroya may affect the efficacy of some medicines work, and some medicines may affect the efficacy of Sogroya ${ }^{3}$.

\section{Sogroya Administration}

- Sogroyais administered exactly as instructed by your health care provider.

- Use Sogroya ${ }^{\circledR} 1$ time each week

- A missed dose of Sogroya ${ }^{\circledR}$, is taken as soon as possible within 3 days (72 hours). Save the missed dose and take the next dose on a scheduled day if more than 3 days (72 hours) have passed.

- Sogroya ${ }^{\circledR}$ stylos are only appropriate for 1 user

- Do not share with another user, even if the needle has changed. You may get infected or another human gets infected from you ${ }^{4}$.

\section{Possible Side Effects}

Sogroya may cause serious side effects, including:

- High mortality rate for people with essential heart or stomach surgery, trauma or extreme breathing difficulties

- Increased risk of cancer or tumour formation, and the risk of cancer relapse increased. Your doctor will need to monitor your cancer or tumour recovery. If you begin to change moles, birthmarks, or skin tone, notify the healthcare provider

- High blood sugar or diabetes or worsening, during treatment with Sogroya (® the blood sugar have to be regulated ${ }^{4}$

- Skull pressure rise. Contact your health care provider if you have headaches, eye infections, nausea or vomiting.

- Extreme reactions to allergy. Get urgent medical attention if you have the following symptoms: swelling of the ears, tongue, mouth or tongue, difficulty breathing, wheezing, extreme itching, skin rashes, redness, or swelling.

- Your body holds too much fluid, such as swelling in your hands or feet, joint or muscle pain, or nerve problems which cause pain, tingling or burning in your hands, arms, legs and feet. Tell your health care provider if you experience any of these fluid retention signs or symptoms

- Drop in the cortisol hormone. The health professional will administer blood tests to track the level of cortisol. Tell your health practitioner if your skin becomes darkened, extreme tired, dizzy, weak or weight loss

- Declining levels of thyroid hormones. The effect of Sogroya can be affected by lower thyroid hormone levels

- Persistent abdominal pain. This could be a pancreatitis symptom. Tale if you have 
fresh abdominal pain with your health care provider

- Loss of fat and tissue weakness in the area of administration of skin where you inject. Talk to your healthcare provider about changing the areas where you inject Sogroya increase in phosphorus, alkaline phosphatase and parathyroid hormone levels in your blood.

The most common side effects of Sogroya may include back pain, joint pain, indigestion, sleep problems, dizziness, swelling of the tonsils, vomiting, high blood pressure, increase in the level of an enzyme in your blood called creatine phosphokinase, weight gain, and low red blood cells

\section{History}

Sogroya, an analogue of human growth hormone, is given one injection per week. The approval was based upon data from multicenter, double-blind, placebo-controlled, active-controlled phase 3 REAL 1 trial which assessed the efficacy and safety of Sologroya in 300 adults who were treatment-naïve with a growth hormone deficit or who had discontinued other formulation of growth hormones for three months.

Patient patients received Sogroya or placebo once weekly or 34 weeks of daily somatropin injections $2: 1: 2$. The key end point was the improvement from the baseline to Week 34 in the percentage of truncal fat.

Results showed that Sogroya treated patients revealed a mean reduction of $1.06 \%$ in truncal fat at week 34 compared with an increase of $0.47 \%$ for placebo (absolute treatment difference of $1.53 \%$; 95\% CI: $-2.68,-0.38 ; P=.009)$. Patients treated with daily somatropin achieved a change in truncal fat $\%$ of $-2.23 \%$ after 34 weeks ${ }^{5}$.

Similar improvements in other clinical end points, including normalized insulin-like growth factor (IGF-I) standard deviation scores at week 34, were observed in the Sogroya and somatropin treatment arms.

\section{Conclusion}

In patients with growth hormone deficiency (GHD) Novo Nordisk published positive Phase II REAL 3 clinical results of somapacitan. A weekly somapacitan-beco, weekly placebo or daily somatropin FDA approved growth hormone was randomly assigned to patients. The efficacy of somapacitan-beco has been calculated by the percentage change of truncular fat that can be associated with metabolic syndrome and other serious problems, regulated by growth hormone.

\section{References}

1. FDA approves weekly therapy for adult growth hormone deficiency. https://www.fda.gov/drugs/drug-safetyand-availability/fda-approves-weeklytherapy-adult-growth-hormone-deficiency. Accessed September 2, 2020.

2. FDA approves once-weekly Sogroya ${ }^{\circledR}$ for the treatment of adult growth hormone deficiency. https://www.novonordiskus.com/media/news-releases.html?122995. Accessed September 2, 2020.

3. Sogroya [package insert]. Plainsboro, $\mathrm{NJ}$ : Novo Nordisk Inc.; 2020.

4. Kargi A, Merriam G. Diagnosis and treatment of growth hormone deficiency in adults. Nat Rev Endocronol. 2013;(9):335345.

5. Brod M, Pohlman B, Hojbjerre L, Adalsteinsson JE, Rasmussen MH. Impact of adult growth hormone deficiency on daily functioning and well-being. BMC Research Notes. 2014;7(813):1-9. 